OU-HET 428

hep-th/0301095

\title{
Intersection Rules for S-Branes
}

\author{
Nobuyoshi Ohta* \\ Department of Physics, Osaka University, Toyonaka, Osaka 560-0043, Japan
}

\begin{abstract}
We give a model-independent derivation of general intersecting rules for spacelike branes (S-branes) in arbitrary dimensions $d$. This is achieved by directly solving bosonic field equations for supergravity coupled to a dilaton and antisymmetric tensor fields with minimal ansätze. We compare the results with those in eleven-dimensional supergravity and other solutions.
\end{abstract}

${ }^{*}$ e-mail address: ohta@phys.sci.osaka-u.ac.jp 
There has been much interest in time-dependent and spacelike brane solutions (Sbranes) of supergravities in eleven and ten dimensions because of its possible connection with tachyon condensations and dS/CFT correspondence $[1,2]$. These theories are the low-energy limits of the string theories and supposedly unifying M-theory of strings. Timedependent solutions are investigated rather recently and not much is known on these solutions. It is thus important to better understand these classical $p$-brane solutions.

The single $p$-brane solutions (Sp-branes) have been discussed in refs. $[1,3,4,5]$ for low-energy effective supergravities (see also refs. [6, 7] for related solutions). Following the usual convention, $\mathrm{S} p$-branes are used for those with $(p+1)$-dimensional Euclidean world-volume. It has then been noted that the more general solutions can be understood as intersecting ones of these fundamental $p$-branes $[8,9]$ and the rules how the branes intersect with each other are given in analogy to the usual branes [10]-[14]. Although the "rules" are consistent with most of the known solutions, it is not clear if there are any other solutions than those given by these rules. The questions we would like to ask here are how general these rules are and how severely they restrict the solutions for supergravities in $d=11$ and lower dimensions.

A systematic approach to formulating the rules for the way how they can intersect has been derived for the usual branes in $[13,14]$. The purpose of this note is to extend this work to the S-branes and clarify what ansätze are really necessary. In particular, we derive the intersection rules from the general approach. We show that the rules are simple consequences of the field equations, which can be easily integrated and the consistency of the solutions reduces the problem of solving the field equations to an algebraic one.

The results of our analysis turn out to be consistent with the superposition rules in ref. [8] for $d=11$ supergravity, but our results apply to more general supergravity coupled to a dilaton and antisymmetric tensors. We show that the requirement that the fields for the each brane be independent is sufficient to give the solutions and intersection rules.

Let us start with the general action for gravity coupled to a dilaton $\phi$ and $m$ different $n_{A}$-form field strengths:

$$
I=\frac{1}{16 \pi G_{d}} \int d^{d} x \sqrt{-g}\left[R-\frac{1}{2}(\partial \phi)^{2}-\sum_{A=1}^{m} \frac{1}{2 n_{A} !} e^{a_{A} \phi} F_{n_{A}}^{2}\right] .
$$

This action describes the bosonic part of $d=11$ or $d=10$ supergravities; we simply drop 
$\phi$ and put $a_{A}=0$ and $n_{A}=4$ for $d=11$, whereas we set $a_{A}=-1$ for the NS-NS 3-form and $a_{A}=\frac{1}{2}\left(5-n_{A}\right)$ for forms coming from the R-R sector. ${ }^{1}$ To describe more general supergravities in lower dimensions, we should include several scalars, but for simplicity we disregard this complication in this paper.

From the action (1), one derives the field equations

$$
\begin{gathered}
R_{\mu \nu}=\frac{1}{2} \partial_{\mu} \phi \partial_{\nu} \phi+\sum_{A} \frac{1}{2 n_{A} !} e^{a_{A} \phi}\left[n_{A}\left(F_{n_{A}}^{2}\right)_{\mu \nu}-\frac{n_{A}-1}{d-2} F_{n_{A}}^{2} g_{\mu \nu}\right], \\
\square \phi=\sum_{A} \frac{a_{A}}{2 n_{A} !} e^{a_{A} \phi} F_{n_{A}}^{2}, \\
\partial_{\mu_{1}}\left(\sqrt{-g} e^{a_{A} \phi} F^{\mu_{1} \cdots \mu_{n_{A}}}\right)=0, \\
\partial_{[\mu} F_{\left.\mu_{1} \cdots \mu_{n_{A}}\right]}=0 .
\end{gathered}
$$

The last equations are the Bianchi identities.

We take the following metric for our system:

$$
d s_{d}^{2}=-e^{2 u_{0}} d t^{2}+\sum_{\alpha=1}^{p} e^{2 u_{\alpha}} d y_{\alpha}^{2}+e^{2 B} d \Sigma_{k, \sigma}^{2},
$$

where $d=p+k+1$, the coordinates $y_{\alpha},(\alpha=1, \ldots, p)$ parametrize the $p$-dimensional world-volume directions and the remaining coordinates of the $d$-dimensional spacetime are the time $t$ and coordinates on $k$-dimensional spherical $(\sigma=+1)$, flat $(\sigma=0)$ or hyperbolic $(\sigma=-1)$ spaces, whose line elements are $d \Sigma_{k, \sigma}^{2}$. Since we are interested in time-dependent solutions, all the functions appearing in the metrics as well as dilaton $\phi$ are assumed to depend only on the time $t$. The Ricci tensors for the metric (3) are

$$
\begin{aligned}
& R_{00}=-\sum_{\alpha=1}^{p}\left[\ddot{u}_{\alpha}+\left(\dot{u}_{\alpha}\right)^{2}-\dot{u}_{\alpha} \dot{u}_{0}\right]-k\left(\ddot{B}+\dot{B}^{2}-\dot{B} \dot{u}_{0}\right), \\
& R_{\alpha \beta}=e^{2\left(u_{\alpha}-u_{0}\right)}\left[\ddot{u}_{\alpha}-\dot{u}_{\alpha} \dot{u}_{0}+\sum_{\gamma=1}^{p} \dot{u}_{\gamma} \dot{u}_{\alpha}+k \dot{B} \dot{u}_{\alpha}\right] \delta_{\alpha \beta}, \\
& R_{a b}=\left\{e^{2\left(B-u_{0}\right)}\left[\ddot{B}+k \dot{B}^{2}-\dot{B} \dot{u}_{0}+\sum_{\alpha=1}^{p} \dot{u}_{\alpha} \dot{B}\right]+\sigma(k-1)\right\} \bar{g}_{a b},
\end{aligned}
$$

\footnotetext{
${ }^{1}$ There may be Chern-Simons terms in the action, but they are irrelevant in our following solutions.
} 
where $\bar{g}_{a b}$ is the metric for the hypersurface $\Sigma_{k, \sigma}$. Here and in what follows, a dot denotes a derivative with respect to $t$.

For the field strengths, we take the most general ones consistent with the field equations and Bianchi identities. The value for an electrically charged Sq-brane (whose worldvolume is $(q+1)$-dimensional) is given by

$$
F_{t \alpha_{1} \cdots \alpha_{q+1}}=\epsilon_{\alpha_{1} \cdots \alpha_{q+1}} \dot{E}, \quad\left(n_{A}=q+2\right),
$$

where $\alpha_{1}, \cdots, \alpha_{q+1}$ stand for the tangential direction to the $\mathrm{S} q$-brane. The magnetic case is given by

$$
F^{\alpha_{q+2} \cdots \alpha_{p} a_{1} \cdots a_{k}}=\frac{1}{\sqrt{-g}} e^{-a \phi} \epsilon^{t \alpha_{q+2} \cdots \alpha_{p} a_{1} \cdots a_{k}} \dot{\tilde{E}}, \quad\left(n_{A}=d-q-2\right)
$$

where $a_{1}, \cdots, a_{k}$ denote the coordinates of the $k$-dimensional hypersurface $\Sigma_{k, \sigma}$. The functions $E$ and $\tilde{E}$ are again assumed to depend only on $t$.

The electric field (5) trivially satisfies the Bianchi identities but the field equations are nontrivial. On the other hand, the field equations are trivial but the Bianchi identities are nontrivial for the magnetic field (6).

We will solve the field eqs. (2) with the simplifying ansatz

$$
-u_{0}+\sum_{\alpha=1}^{p} u_{\alpha}+k B=0
$$

which simplifies the field equations (2) considerably. For both cases of electric (5) and magnetic (6) fields, we find that the field eqs. (2) are cast into

$$
\begin{aligned}
& -\ddot{u}_{0}+\left(\dot{u}_{0}\right)^{2}-\sum_{\alpha=1}^{p}\left(\dot{u}_{\alpha}\right)^{2}-k \dot{B}^{2}=\frac{1}{2} \dot{\phi}^{2}+\sum_{A} \frac{d-q_{A}-3}{2(d-2)} S_{A}\left(\dot{E}_{A}\right)^{2}, \\
& \ddot{u}_{\alpha}=-\sum_{A} \frac{\delta_{A}^{(\alpha)}}{2(d-2)} S_{A}\left(\dot{E}_{A}\right)^{2}, \quad(\alpha=1, \cdots, p), \\
& \ddot{B}+\sigma(k-1) e^{2 u_{0}-2 B}=\sum_{A} \frac{q_{A}+1}{2(d-2)} S_{A}\left(\dot{E}_{A}\right)^{2}, \\
& \ddot{\phi}=\sum_{A} \frac{\epsilon_{A} a_{A}}{2} S_{A}\left(\dot{E}_{A}\right)^{2}, \\
& \left(S_{A} \dot{E}_{A}\right)=0
\end{aligned}
$$


where $A$ denotes the kinds of $q_{A}$-branes and we have defined

$$
S_{A} \equiv \exp \left(\epsilon_{A} a_{A} \phi-2 \sum_{\alpha \in q_{A}} u_{\alpha}\right)
$$

and

$$
\delta_{A}^{(\alpha)}=\left\{\begin{array} { l } 
{ d - q _ { A } - 3 } \\
{ - ( q _ { A } + 1 ) }
\end{array} \quad \text { for } \left\{\begin{array}{l}
y_{\alpha} \text { belonging to } q_{A} \text {-brane } \\
\text { otherwise }
\end{array}\right.\right.
$$

and $\epsilon_{A}=+1(-1)$ corresponds to electric (magnetic) fields. For magnetic case we have dropped the tilde from $E_{A}$. Equations (8), (9) and (10) are the 00, $\alpha \alpha$ and $a b$ components of the Einstein equation in (2), respectively. The last one is the field equation for the field strengths of the electric fields and/or Bianchi identity for the magnetic ones. It is remarkable that both the electric and magnetic cases can be treated simultaneously just by using the sign $\epsilon_{A}$. This is because the original system (1) has the S-duality symmetry under

$$
g_{\mu \nu} \rightarrow g_{\mu \nu}, \quad F_{n_{A}} \rightarrow e^{-a_{A} \phi} * F_{n_{A}}, \quad \phi \rightarrow-\phi .
$$

From eq. (12) one finds

$$
S_{A} \dot{E}_{A}=c_{A},
$$

where $c_{A}$ is a constant. With the help of eq. (16), we find that eqs. (9) and (11) give

$$
\begin{aligned}
\dot{u}_{\alpha} & =-\sum_{A} \frac{\delta_{A}^{(\alpha)}}{2(d-2)} c_{A} E_{A}+c_{\alpha}, \\
\dot{\phi} & =\sum_{A} \frac{\epsilon_{A} a_{A}}{2} c_{A} E_{A}+c_{\phi},
\end{aligned}
$$

where $c_{\alpha}$ and $c_{\phi}$ are integration constants. Let us next define

$$
g(t)=\left(u_{0}-B\right) /(k-1)
$$

We find from (7)

$$
B=g-\frac{1}{k-1} \sum_{\alpha=1}^{p} u_{\alpha}, \quad u_{0}=k g-\frac{1}{k-1} \sum_{\alpha=1}^{p} u_{\alpha}
$$


Using (17), we get

$$
\begin{gathered}
\dot{B}=\dot{g}+\sum_{A} \frac{q_{A}+1}{2(d-2)} c_{A} E_{A}-\frac{1}{k-1} \sum_{\alpha=1}^{p} c_{\alpha}, \\
\dot{u}_{0}=k \dot{g}+\sum_{A} \frac{q_{A}+1}{2(d-2)} c_{A} E_{A}-\frac{1}{k-1} \sum_{\alpha=1}^{p} c_{\alpha},
\end{gathered}
$$

Substituting (16) and (20) into (10), we obtain

$$
\ddot{g}+\sigma(k-1) e^{2(k-1) g}=0,
$$

which yields

$$
\dot{g}^{2}+\sigma e^{2(k-1) g}=\beta^{2},
$$

where $\beta$ is an integration constant. The solution to eq. (23) is given by

$$
g(t)= \begin{cases}\frac{1}{k-1} \ln \frac{\beta}{\cosh \left[(k-1) \beta\left(t-t_{1}\right)\right]} & : \sigma=+1, \\ \pm \beta\left(t-t_{1}\right) & : \sigma=0, \\ \frac{1}{k-1} \ln \frac{\beta}{\sinh \left[(k-1) \beta\left(t-t_{1}\right)\right]} & : \sigma=-1,\end{cases}
$$

where $t_{1}$ is another integration constant.

Substituting eqs. (17), (22) and (23) into (8) yields

$$
\begin{aligned}
& (k-1)\left(\sum_{A} \frac{q_{A}+1}{2(d-2)} c_{A} E_{A}-\frac{1}{k-1} \sum_{\alpha=1}^{p} c_{\alpha}\right)^{2}+\sum_{\alpha=1}^{p}\left(\sum_{A} \frac{\delta_{A}^{(\alpha)}}{2(d-2)} c_{A} E_{A}-c_{\alpha}\right)^{2} \\
& +\frac{1}{2}\left(\sum_{A} \frac{\epsilon_{A} a_{A}}{2} c_{A} E_{A}+c_{\phi}\right)^{2}+\sum_{A} \frac{c_{A}}{2} \dot{E}_{A}-k(k-1) \beta^{2}=0
\end{aligned}
$$

This equation must be valid for arbitrary functions $E_{A}$ of $t$. From the $E_{A}$-independent part of eq. (25), one finds

$$
\frac{1}{k-1}\left(\sum_{\alpha=1}^{p} c_{\alpha}\right)^{2}+\sum_{\alpha=1}^{p} c_{\alpha}^{2}+\frac{1}{2} c_{\phi}^{2}=k(k-1) \beta^{2} .
$$

We can then rewrite eq. (25) as

$$
\sum_{A, B}\left[M_{A B} \frac{c_{A}}{2}-\delta_{A B}\left\{\left(\frac{1}{E_{A}}\right)+\frac{2 \tilde{c}_{A}}{E_{A}}\right\}\right] \frac{c_{B}}{2} E_{A} E_{B}=0,
$$


where

$$
\begin{aligned}
M_{A B} & =\sum_{\alpha=1}^{p} \frac{\delta_{A}^{(\alpha)} \delta_{B}^{(\alpha)}}{(d-2)^{2}}+(k-1) \frac{\left(q_{A}+1\right)\left(q_{B}+1\right)}{(d-2)^{2}}+\frac{1}{2} \epsilon_{A} a_{A} \epsilon_{B} a_{B}, \\
\tilde{c}_{A} & =\sum_{\alpha \in q_{A}} c_{\alpha}-\frac{1}{2} c_{\phi} \epsilon_{A} a_{A} .
\end{aligned}
$$

Since $M_{A B}$ is constant, eq. (27) cannot be satisfied for arbitrary functions $E_{A}$ of $t$ unless the second term inside the square bracket is a constant. Requiring this to be a constant tells us that the function $E_{A}$ must satisfy

$$
\left(\frac{1}{E_{A}}\right)+\frac{2 \tilde{c}_{A}}{E_{A}}=\tilde{c}_{A} N_{A},
$$

or

$$
E_{A}=\frac{e^{\tilde{c}_{A}\left(t-t_{A}\right)}}{N_{A} \cosh \tilde{c}_{A}\left(t-t_{A}\right)},
$$

where $N_{A}$ is a normalization factor and $t_{A}$ is an integration constant. In this way, the problem reduces to the algebraic equation (27) supplemented by (30) without making any assumption other than (7).

Equation (27) has two implications if we take independent functions for the fields $E_{A}$. In this case, first putting $A=B$ in eq. (27), we learn that

$$
c_{A}=\frac{2(d-2) \tilde{c}_{A} N_{A}}{\Delta_{A}},
$$

where

$$
\Delta_{A}=\left(q_{A}+1\right)\left(d-q_{A}-3\right)+\frac{1}{2} a_{A}^{2}(d-2) .
$$

By use of eqs. (31) and (32), eqs. (17), (20) and (21) can be integrated with the results

$$
\begin{aligned}
u_{0} & =k g(t)+\sum_{A} \frac{q_{A}+1}{\Delta_{A}} \ln \cosh \tilde{c}_{A}\left(t-t_{A}\right)+c_{0} t+c_{0}^{\prime}, \\
u_{\alpha} & =-\sum_{A} \frac{\delta_{A}^{(\alpha)}}{\Delta_{A}} \ln \cosh \tilde{c}_{A}\left(t-t_{A}\right)+\tilde{c}_{\alpha} t+c_{\alpha}^{\prime}, \\
B & =g(t)+\sum_{A} \frac{q_{A}+1}{\Delta_{A}} \ln \cosh \tilde{c}_{A}\left(t-t_{A}\right)+c_{0} t+c_{0}^{\prime}, \\
\phi & =\sum_{A} \frac{(d-2) \epsilon_{A} a_{A}}{\Delta_{A}} \ln \cosh \tilde{c}_{A}\left(t-t_{A}\right)+\tilde{c}_{\phi} t+c_{\phi}^{\prime},
\end{aligned}
$$


where $c_{\alpha}^{\prime}$ 's are new integration constants and

$$
\begin{aligned}
& c_{0}=\sum_{A} \frac{q_{A}+1}{\Delta_{A}} \tilde{c}_{A}-\frac{\sum_{\alpha=1}^{p} c_{\alpha}}{k-1}, \quad c_{0}^{\prime}=-\frac{\sum_{\alpha=1}^{p} c_{\alpha}^{\prime}}{k-1}, \quad \tilde{c}_{\alpha}=c_{\alpha}-\sum_{A} \frac{\delta_{A}^{(\alpha)}}{\Delta_{A}} \tilde{c}_{A}, \\
& \tilde{c}_{\phi}=c_{\phi}+\sum_{A} \frac{(d-2) \epsilon_{A} a_{A}}{\Delta_{A}} \tilde{c}_{A} .
\end{aligned}
$$

To fix the normalization $N_{A}$, we go back to eq. (13). Using (34), we find

$$
S_{A}=\left[\cosh \tilde{c}_{A}\left(t-t_{A}\right)\right]^{2} e^{\epsilon_{A} a_{A} c_{\phi}^{\prime}-2 \sum_{\alpha \in q_{A}} c_{\alpha}^{\prime}}
$$

which, together with (16) and (32), leads to

$$
N_{A}=\sqrt{\frac{\Delta_{A}}{2(d-2)}} e^{\epsilon_{A} a_{A} c_{\phi}^{\prime} / 2-\sum_{\alpha \in q_{A}} c_{\alpha}^{\prime}} .
$$

Our metric and other fields are thus finally given by

$$
\begin{gathered}
d s_{d}^{2}=\prod_{A}\left[\cosh \tilde{c}_{A}\left(t-t_{A}\right)\right]^{2 \frac{q_{A}+1}{\Delta_{A}}}\left[e^{2 k g(t)+2 c_{0} t+2 c_{0}^{\prime}}\left\{-d t^{2}+e^{-2(k-1) g(t)} d \Sigma_{k, \sigma}^{2}\right\}\right. \\
\left.+\sum_{\alpha=1}^{p} \prod_{A}\left[\cosh \tilde{c}_{A}\left(t-t_{A}\right)\right]^{-2 \frac{\gamma_{A}^{(\alpha)}}{\Delta_{A}}} e^{2 \tilde{c}_{\alpha} t+2 c_{\alpha}^{\prime}} d y_{\alpha}^{2}\right], \\
E_{A}=\frac{e^{\tilde{c}_{A}\left(t-t_{A}\right)}}{N_{A} \cosh \tilde{c}_{A}\left(t-t_{A}\right)}, \quad \tilde{c}_{A}=\sum_{\alpha \in q_{A}} c_{\alpha}-\frac{1}{2} c_{\phi} \epsilon_{A} a_{A} .
\end{gathered}
$$

where we have defined

$$
\gamma_{A}^{(\alpha)}=\left\{\begin{array} { l } 
{ d - 2 } \\
{ 0 }
\end{array} \text { for } \left\{\begin{array}{l}
y_{\alpha} \text { belonging to } q_{A} \text {-brane } \\
\text { otherwise }
\end{array}\right.\right.
$$

These solutions contain $2 p+2$ integration constants $c_{\alpha}, c_{\alpha}^{\prime}(\alpha=1, \cdots, p), c_{\phi}, c_{\phi}^{\prime}$, and $t_{1}$ and $t_{A}$ with $\beta$ determined by eq. (26). Among these, $c_{\alpha}^{\prime}$ can be removed by rescaling the coordinates, and $t_{1}$ by a shift of the time. Without any preference of the choice of other paremeters, we leave these as free parameters. Thus the general solutions can be constructed by the following rules: (1) All the directions are multiplied by $\left[\cosh \tilde{c}_{A}\left(t-t_{A}\right)\right]^{2 \frac{q_{A}+1}{\Delta_{A}}}$, and in addition, (2) the overall transverse direction (time and $k$-dimensional space) has the form $e^{2 c_{0} t}\left[-e^{2 k g(t)} d t^{2}+e^{2 g(t)} d \Sigma_{k, \sigma}^{2}\right]$ up to the rescaling of the coordinates, (3) the coordinates belonging to the brane are multiplied by $\left[\cosh \tilde{c}_{A}\left(t-t_{A}\right)\right]^{-2 \frac{d-2}{\Delta_{A}}}$. When these are specified 
to $d=11$ supergravity and the integration constants are chosen appropriately, these give the superposition rules discussed in ref. [8].

The solution given in ref. [3] is reproduced if we restrict these to a single S-brane and choose the integration constants as

$$
\begin{aligned}
& c_{\gamma}=\alpha-\frac{a}{\chi} c_{1},(\gamma=1, \cdots, p) ; \quad c_{\gamma}=-\frac{p}{q-1}\left(\alpha-\frac{a}{\chi} c_{1}\right),(\gamma=p+1, \cdots, p+q-k) \\
& c_{\phi}=\frac{d-2}{q-1} a \alpha+\frac{2 p}{\chi} c_{1} ; \quad c_{\gamma}^{\prime}=\frac{1}{\chi}\left(\ln \frac{(d-2) \chi \alpha^{2}}{(q-1) b^{2}}-a c_{2}\right),(\gamma=1, \cdots, p) \\
& c_{0}^{\prime}=c_{b}^{\prime}=c_{\gamma}^{\prime}=-\frac{p}{\chi(q-1)}\left(\ln \frac{(d-2) \chi \alpha^{2}}{(q-1) b^{2}}-a c_{2}\right),(\gamma=p+1, \cdots, p+q-k)
\end{aligned}
$$

with $q \rightarrow p-1, p \rightarrow p+q-k, \epsilon=-1$ and $\chi=2 p+(d-2) a^{2} /(q-1) .^{2}$ Equation (26) reduces to

$$
\frac{p c_{1}^{2}}{\chi}+\frac{(d-2) \chi \alpha^{2}}{2(q-1)}-k(k-1) \beta^{2}=0,
$$

and the normalization is determined to be

$$
N_{A}=\frac{(q-1) b}{2(d-2) \alpha}
$$

in complete agreement with ref. [3].

The second condition following from eqs. (27) is $M_{A B}=0$ for $A \neq B$. This leads to the intersection rules for two branes: If $q_{A}$-brane and $q_{B}$-brane intersect over $\bar{q}\left(\leq q_{A}, q_{B}\right)$ dimensions, this gives

$$
\bar{q}=\frac{\left(q_{A}+1\right)\left(q_{B}+1\right)}{d-2}-1-\frac{1}{2} \epsilon_{A} a_{A} \epsilon_{B} a_{B}
$$

Remember that the world-volume of $q$-branes lies in $(q+1)$-dimensional space and not in time. For eleven-dimensional supergravity, we have electric S2-branes, magnetic S5branes and no dilaton $a_{A}=0$. The rule (43) tells us that S2-brane can intersect with S2-brane over a '0-brane' $(\bar{q}=0)$ (which actually lives in 1-dimensional space) and with S5-brane over a 'string' $(\bar{q}=1)$ (2-dimensional space), and S5-brane can intersect with S5-brane over '3-brane' $(\bar{q}=3)$ (4-dimensional space), again in agreement with refs. [8]. In particular, our results show that there is no other intersecting solution as long as we

\footnotetext{
${ }^{2}$ Here $c_{1}$ and $c_{2}$ on the rhs are those used in ref. [3] and should not be confused with our $c_{\gamma},(\gamma=1,2)$.
} 
treat the functions $E_{A}$ with different index $A$ as independent. If this condition is relaxed, there may be other solutions.

For all the spacelike $\mathrm{D} q$-brane solutions in type II superstrings, we find

$$
\epsilon a=\frac{3-q}{2},
$$

which tells us that the intersection rule is

$$
\bar{q}=\frac{q_{A}+q_{B}}{2}-2 .
$$

These solutions do not preserve any supersymmetry. In fact, they are supposed to correspond to branes with Dirichlet boundary conditions in the time direction, and hence describe configurations which exist only for a fixed time. These also contain singularity somewhere in time. Closer examination of their properties would be quite interesting.

We note that our derivation is a simple generalization of the general method developed in ref. [14]. It is quite satisfying to see that this is so useful method. There are also an important class of time-dependent solutions called null-branes $[15,16]$, which preserve supersymmetry. It would be also interesting to apply our method to these solutions.

To summarize, we have given quite a general model-independent derivation of the superposition rules in arbitrary dimensions. The intersection rules simply follow from the field equations if we require that the functions $E_{A}$ with different index $A$ be independent. In all cases, the algebraic eq. (27) (together with (30)) must be satisfied, and this equation should be most useful to examine possible solutions. We hope to discuss various properties of these solutions using the hints from dualities implied by underlying string dynamics elsewhere.

\section{Acknowledgement}

This work was supported in part by Grants-in-Aid for Scientific Research Nos. 12640270 and 02041 .

\section{References}

[1] M. Gutperle and A. Strominger, JHEP 0204 (2002) 018, hep-th/0202210. 
[2] A. Strominger, JHEP 0110 (2001) 034, hep-th/0106113.

[3] C.M. Chen, D.M. Gal'tsov and M. Gutperle, Phys. Rev. D 66 (2002) 024043, hepth/0204071.

[4] M. Kruczenski, R.C. Myers and A.W. Peet, JHEP 0205 (2002) 039, hep-th/0204144.

[5] S. Roy, JHEP 0208 (2002) 025, hep-th/0205198.

[6] K. Behrndt and S. Förste, Nucl. Phys. B 430 (1994), 441, hep-th/9403179;

H. Lu, S. Mukherji, C.N. Pope and K.-W. Xu, Phys. Rev. D 55 (1997) 7926, hepth/9610107;

A. Lukas, B.A. Ovrut and D. Waldram, Nucl. Phys. B 495 (1997) 365, hepth/9610238;

C. Grojean, F. Quevedo, I. Zavala and G. Tasinato, JHEP 0108(2001) 005, hepth/0106120.

[7] C.M. Hull, JHEP 9807 (1998) 021, hep-th/9806146.

[8] N.S. Deger and A. Kaya, JHEP 0207 (2002) 038, hep-th/0206057.

[9] V.D. Ivashchuk and V.N. Melnikov, J. Math. Phys. 39 (1998) 2866, hep-th/9708157; V.D. Ivashchuk, Class. Quant. Grav. 20 (2003) 261, hep-th/0208101.

[10] G. Papadopoulos and P. Townsend, Phys. Lett. B 380 (1996) 273, hep-th/9603087.

[11] A. A. Tseytlin, Nucl. Phys. B 475 (1996)149, hep-th/9604035.

[12] J. P. Gauntlett, D. A. Kastor and J. Traschen, Nucl. Phys. B 478 (1996) 544, hepth/9604179.

[13] R. Argurio, F. Englert and L. Houart, Phys. Lett. B 398 (1997) 61, hep-th/9701042.

[14] N. Ohta, Phys. Lett. B 403 (1997) 218, hep-th/9702164.

[15] I.I. Kogan and N.B. Reis, Int. Jour. Mod. Phys. A16 (2001) 4567, hep-th/0107163.

[16] J. Figueroa-O'Farrill and J. Simon, JHEP 0112 (2001) 011, hep-th/0110170. 